\title{
Single-cell RNA-seq of the Developing Cardiac Outflow Tract Reveals Convergent Development of the Vascular Smooth Muscle Cells at the Base of the Great Arteries
}

\author{
Xuanyu Liu ${ }^{1}$, Wen Chen ${ }^{1}$, Wenke Li ${ }^{1}$, James R. Priest ${ }^{2}$, Jikui Wang ${ }^{3}$, Zhou Zhou ${ }^{1}$ \\ 1. State Key Laboratory of Cardiovascular Disease, Beijing Key Laboratory for Molecular \\ Diagnostics of Cardiovascular Diseases, Center of Laboratory Medicine, Fuwai Hospital, \\ National Center for Cardiovascular Diseases, Chinese Academy of Medical Sciences and \\ Peking Union Medical College, Beijing 100037, China \\ 2. Stanford University School of Medicine, Stanford, CA 94305, USA \\ 3. Henan Key Laboratory for Medical Tissue Regeneration, School of Basic Medical Sciences, \\ Xinxiang Medical University. Xinxiang 453003, China
}

Running title: Single-cell RNA-seq of the developing cardiac outflow tract

\section{Subject Terms:}

Developmental Biology, Vascular Biology, Gene Expression and Regulation, Congenital Heart Disease

\section{Address correspondence to:}

Dr. Jikui Wang

School of Basic Medical Sciences

Xinxiang Medical University

No. 601, Jinsui Road

453003 Xinxiang

China

Tel: +8618336067869

Fax:+86 010-88398055

wangjikui00@gmail.com
Dr. Zhou Zhou

Fuwai Hospital

Chinese Academy of Medical Sciences

No. 167 Beilishi Street, Xicheng District 100037 Beijing

China

Tel: +86 010-88398055

Fax:+86 010-88398055

zhouzhou@fuwaihospital.org 


\section{ABSTRACT}

Rationale: Cardiac outflow tract (OFT) is a major hotspot for congenital heart diseases (CHDs). A thorough understanding of the cellular diversity, transitions and regulatory networks of normal OFT development is essential to decipher the etiology of OFT malformations.

Objective: We sought to explore the cellular diversity and transitions between cell lineages during OFT development.

Methods and Results: We performed single-cell transcriptomic sequencing of 55,611 mouse OFT cells from three developmental stages that generally correspond to the early, middle and late stages of OFT remodeling and septation. We identified 17 cell clusters that could be assigned to six cell lineages. Among these lineages, the macrophage and VSMC lineages of the developing OFT have seldom been previously described. Known cellular transitions, such as endothelial to mesenchymal transition, have been recapitulated. In particular, we identified convergent development of the VSMC lineage, where intermediate cell subpopulations were found to be involved in either myocardial to VSMC trans-differentiation or mesenchymal to VSMC transition. Through single-molecule in situ hybridization, we observed that cells expressing the myocardial marker Myh7 co-expressed the VSMC marker gene Cxcl12 in OFT walls, thus confirming the existence of myocardial to VSMC trans-differentiation. Moreover, we found that the Penk $k^{+}$cluster c8, a relatively small mesenchymal subpopulation that was undergoing mesenchymal to VSMC transition, was associated with the fusion of OFT cushions. We also uncovered the expression dynamics and critical transcriptional regulators potentially governing cell state transitions. Finally, we developed web-based interactive interfaces to facilitate further data exploration.

Conclusions: We provide a single-cell reference map of cell states for normal OFT development, which will be a valuable resource for the CHD community. Our data support the existence of myocardial to VSMC trans-differentiation and convergent development of the VSMC lineage at the base of the great arteries.

\section{Keywords:}

cardiac outflow tract; single-cell RNA-seq; trans-differentiation; vascular smooth muscle; convergent development

\section{Nonstandard Abbreviations and Acronyms:}

\section{EndoMT endothelial to mesenchymal transition}

GRN gene regulatory network

KNN k nearest neighbor

OFT outflow tract

SHF second heart field

UMI unique molecular identifier

VSMC vascular smooth muscle cell 


\section{INTRODUCTION}

Congenital heart disease (CHD) is the most common form of human birth defects ( $\sim 1 \%$ of live births) and represents the leading cause of mortality from birth defects worldwide ${ }^{1}$. Approximately $30 \%$ of CHDs involve abnormalities in cardiac outflow tract (OFT) development, thus constituting a large class of CHDs, namely OFT malformations, such as persistent truncus arteriosus (PTA), double outlet right ventricle (DORV), transposition of the great arteries (TGA) and aortopulmonary-window (APW $)^{2,3}$. It is therefore acknowledged that OFT is a major hotspot for human $\mathrm{CHDs}^{4}$. OFT malformations require surgical repair once diagnosed and usually have a poor prognosis ${ }^{5}$. However, the etiology for the majority of this severe class of CHDs remains unknown.

Cardiac OFT is a transient conduit during embryogenesis at the arterial pole of the heart, connecting the aortic sac with embryonic ventricles, which undergoes rotation and septation (a.k.a., OFT remodeling) to give rise to the base of the pulmonary trunk and ascending aorta; thus, this process is critical for the establishment of separate systemic and pulmonary circulations $^{6}$. The high incidence of OFT malformations may be explained by the complexity of OFT development, which requires intricate interplay and transitions among diverse cell populations, including cardiac cells and migrating extra-cardiac cells, making it particularly susceptible to genetic or environmental perturbations. A thorough understanding of the cellular diversity, cellular transitions and regulatory networks of normal OFT development is essential to decipher the etiology of OFT malformations.

Multiple disparate cell types have been implicated in OFT development through tightly coordinated processes such as migration, differentiation and transition. Initially, the OFT wall basically consists of a solitary tube of myocardium derived from the second heart field (SHF) ${ }^{7}$. At the initiation of remodeling, the interstitial space between the myocardium and endothelium is filled with extracellular matrix ("cardiac jelly") secreted by mesenchymal cells that form OFT cushions at the proximal and distal regions ${ }^{8}$. Cardiac neural crest cells (CNCCs) migrate into the cardiac OFT, where they first join the mesenchyme of the distal and then the proximal cushions, playing an essential role in the fusion of the distal cushions to form a smooth muscle septum, i.e., aorticopulmonary septum, which divides the aorta and pulmonary trunk ${ }^{9,}{ }^{10}$. In addition to CNCCs, distal OFT cushions are colonized by cells derived from the SHF, which eventually give rise to smooth muscle walls of the base of the great arteries ${ }^{11}$. In contrast, proximal OFT cushions are mainly populated by the mesenchymal progenies of OFT endothelial cells that undergo endothelial to mesenchymal transition (EndoMT) ${ }^{12}$. Besides, the OFT remodeling is accompanied by other biological processes, for example, the maturation of the smooth muscle walls, since the OFT wall changes from a myocardial to an arterial phenotype with development ${ }^{13}$. Given the complexity of OFT development, we expect a heterogeneous cellular composition represented by multiple subpopulations of the same cell type and extensive cellular transitions occurring between different cell types. However, the cell type and cell states of the cardiac OFT during development have not yet been systematically dissected. 
Recent technical advances have enabled the transcriptomes of tens of thousands of cells to be assayed at single-cell resolution in a single experiment ${ }^{14}$. Single-cell RNA-seq has shown itself to be a powerful tool to provide insights into the processes underlying developmental, physiological and disease systems ${ }^{15}$. Single-cell RNA-seq enables the dissection of cellular heterogeneity in an unbiased manner with no need for any prior knowledge of the cell population ${ }^{16}$. Unsupervised clustering of cells based on genome-wide expression profiles enables the identification of novel cell types or subpopulations, as well as gene signatures for all cell types. Beyond cellular heterogeneity dissection, single-cell RNA-seq data empower systematic interrogations of the developmental trajectory of cell lineages in tissue systems and the regulatory networks underlying the cell state transition processes ${ }^{17}$. Although traditional gene knockdown studies have uncovered regulators during OFT development ${ }^{10}$, how genes are regulated under a normal developmental context remains unclear. Single-cell RNA-seq has been applied to study the cellular diversity of embryonic ${ }^{18,19}$ or adult heart ${ }^{20,21}$ at the whole organ level; however, few or a limited number of cardiac OFT cells have been sampled in these studies.

Here, we performed single-cell transcriptomic sequencing of 55,611 mouse OFT cells from three successive developmental stages corresponding to the early, middle and late stages of OFT remodeling and septation. We sought to unbiasedly and systematically dissect the cell types and states during OFT development. We explored the cell lineage relationships and cellular state transitions during OFT development, as well as the critical transcription regulators underlying the transitions. We identified convergent development of the vascular smooth muscle cells (VSMCs) at the base of the great arteries, where intermediate cell subpopulations were found to be involved in either myocardial to VSMC trans-differentiation or mesenchymal to VSMC transition. Our study provided a single-cell reference map of cell transcriptomic states for OFT normal development.

\section{METHODS}

\section{See Online Methods.}

The sequencing read data have been deposited in Genome Sequence Archive (GSA; http://gsa.big.ac.cn/) and are accessible through accession number CRA001120.

\section{RESULTS}

\section{Single-cell transcriptomic sequencing and unbiased clustering of developing OFT cells.}

To obtain a map of the cellulome for the developing OFT during remodeling and septation, we isolated and sequenced a total of 64,605 cells from three successive developmental stages, 
namely, ps47 (47 pairs of somites), ps49 and ps51, which generally correspond to the early (initiation), middle and late (almost completion) stages of septation, respectively (Figure 1A). Sequencing quality metrics were similar across samples, reflecting little technical variation among samples (Online Table I). After stringent quality filtering and discarding a small number (297) of red blood cells, we obtained a high-quality transcriptomic dataset for 55,611 cells.

Based on the single-cell transcriptional profiles, unsupervised clustering identified 17 cell clusters at the chosen resolution (Figure 1B) that represent distinct cell types or cell subpopulations. Next, we compared the relative proportions of cells from different samples in each cluster (Figure 1C). Importantly, there was no significant difference in cell fraction between the two biological replicates for each stage, reflecting the validity of cell clustering (Wilcoxon signed rank test $\mathrm{P}$-value $=0.25$ ). Moreover, all the clusters contained cells from the three stages except c14, a small cluster (342 cells), suggesting that the samples for each stage covered all common cell states throughout development. However, the relative proportions varied greatly between stages, as reflected by the observation that the cell fractions for some clusters, e.g., c11, remarkably changed during development (Figure 1C).

\section{Cellular diversity and heterogeneity during OFT development identified by single-} cell transcriptomic analysis.

To define the identity of each cell cluster, we performed differential expression analysis between each cluster and all others (Online Table II), and assigned a specific cell type to each cluster based on the established lineage-specific marker genes (Figure 2A). Cluster c15 represented a small group of macrophages that resided in the developing OFT as the cells in this cluster specifically expressed Fcgrl and Adgre ${ }^{21,22}$. Clusters c10 and c11 constituted the epicardial lineage as they specifically expressed $U p k 3 b$ and $U p k 1 b^{19,23}$. Clusters c5, c6 and c12 highly expressed Ecscr and $C d h 5^{19,24}$; thus, they belonged to the endocardial lineage. Clusters c2, c9, c14 and c16 highly expressed myocardial marker genes, such as Myh7 and Myl4 ${ }^{19,25}$. The mesenchymal lineage comprised four closely aligned clusters, namely, $\mathrm{c} 0, \mathrm{c} 1, \mathrm{c} 7$ and $\mathrm{c} 8$, which highly expressed mesenchymal marker genes, such as Postn and Cthrcl ${ }^{19}$. The VSMC lineage included c3, c4 and c13, which specifically expressed $\operatorname{Rgs} 5$, a gene that is abundantly expressed in arterial smooth muscle cells ${ }^{26,27}$, and Cxcl12, a chemokine encoding gene that is highly expressed in the walls of the aorta and pulmonary trunk of the embryonic heart $(\mathrm{E} 12.5)^{28}$. We also assessed the expression intensity distribution of contractile markers for smooth muscle cells, including Acta 2, Tagln, Cnnl and Myl9 $9^{25,29}$. We observed the expression of these markers in our VSMC lineage clusters. However, the specificity of these markers was not ideal since they were also highly expressed in myocardial and mesenchymal lineages (Online Figure IA), which is in line with previous knowledge ${ }^{29}$. Moreover, only a small group of cells expressed Myh11, the most specific contractile marker for mature $\mathrm{VSMCs}^{29}$, and clustered on the edge of c3, which may represent a group of relatively mature VSMCs. By contrast, the expression of contractile markers for embryonic myocardial cells ${ }^{25}$, including Myh7, Tnnc1, Tnnt2, Myl2 and 
Myl4, were relatively specific in myocardial lineage clusters (Online Figure IB). Ultimately, we identified clusters of VSMCs at the base of the great arteries, most of which may exhibit an immature, synthetic phenotype at this developmental stage.

The six cell lineages identified by established marker genes were further confirmed by hierarchical clustering analysis, which showed that the clusters assigned to the same lineage were grouped together and closely aligned on the tree (Figure 2B). Once cell identity was assigned, we explored the relative proportions of each cell lineage during development (Figure 2B \& 2C). The mesenchymal lineage in OFT cushions constituted the most abundant cell type $(46.3 \%)$, and the relative proportion significantly decreased at the late stage (Student's t-Test Pvalue $<0.05$ ), suggesting that an active cellular transition occurred at the late stage. The macrophages accounted for only $0.5 \%$ of all cells, and the relative proportion did not significantly change during development. Strikingly, the myocardial lineage diminished over time, while the VSMC lineage expanded during development, in accordance with the myocardial to arterial phenotypic change. The epicardial lineage also significantly expanded with development, consistent with a previous report ${ }^{19}$.

\section{Machine learning-based selection of molecular signatures for cell lineages and clusters during OFT development.}

To select molecular signatures that define the identified cell lineages and clusters, we adopted a machine learning-based strategy (Online Figure II). All the random forest models we trained achieved a good classification performance (AUC range: 0.94-1; Online Figure III \& Online Figure IV). The top genes that contributed the most to the models were selected as the molecular signatures for each of the six cell lineages or each subpopulation/cluster (Figure 2D). For cell lineages, most selected genes have been reported to be specifically expressed in a cell type. For example, $W t 1$ in epicardium ${ }^{30}$, Ccan in cushion mesenchyme ${ }^{31}, M y h 7$ in myocardium ${ }^{32}$, Rasip 1 in endocardium ${ }^{33}$ and Fbln 5 in $\mathrm{VSMCs}^{34}$. However, some genes have seldom been previously described to be expressed specifically in a cell lineage of the developing OFT, e.g., Aldh1a2 in epicardium and Papss 2 in mesenchyme. For clusters of each lineage, some selected markers have been reported to be cell type-specific in the embryonic heart $(\mathrm{E} 10.5)^{19}$; however, they were found to be expressed in a cluster-specific manner. For example, Tmem255a was reported to be the epicardium marker of the E10.5 heart ${ }^{19}$; however, in the present study, it was specifically expressed in only one of the two subpopulations of the epicardial cells, namely, c10 (Online Figure IV).

\section{Convergent development of the VSMCs at the base of the great arteries inferred from}

\section{a KNN graph.}

To infer the relationships of cell lineages, we visualized the single-cell dataset using a force- 
directed layout of a k-nearest-neighbor (KNN) graph (Figure 3A \& 3B), which has been proven to perform better than t-distributed stochastic neighbor embedding (tSNE) for visualizing complex and continuous gene expression topologies of cell populations ${ }^{35}$. Cell clusters of the same lineage were closely aligned in the KNN graph, and known relationships among lineages have been well recapitulated. For example, the EndoMT process was reflected by an abundance of potentially intermediate, transitioning cells connecting the endocardial lineage (c5) and mesenchymal lineage (c1), while these cell clusters were separated in the tSNE plot shown in Figure 1B. The dynamic changes in cell states during development could be well visualized when the cells were displayed by developmental stage (Figure 3C). For example, we observed the rapidly diminished myocardium and the expanded epicardium.

In particular, we noticed five cell clusters, including c1, c3, c4, c8 and c9, which may be directly related to the development of VSMCs (Figure 3D \& 3E). VSMC cluster c4 expanded during the early and middle stage and was almost replaced by VSMC cluster $\mathrm{c} 3$ at the late stage. In the KNN plot (Figure 3D), c4 became closer to $\mathrm{c} 3$ over time. Therefore, we speculated that $\mathrm{c} 4$ may represent an intermediate state and c3 a more mature state of VSMCs. Intriguingly, myocardial subpopulation c9 and VSMC progenitor population c4 were densely connected, and a considerable number of cells were in between, which may represent intermediate, transitioning cell states. Therefore, this finding could imply that myocardial to VSMC trans-differentiation may occur during OFT development. Moreover, a relatively small (1,924 cells) mesenchymal cluster, $\mathrm{c} 8$, was closely aligned with $\mathrm{c} 4$ and became closer to $\mathrm{c} 4$ over time, implying that $\mathrm{c} 8$ may represent a special mesenchymal subpopulation actively involved in the mesenchymal to VSMC transition at a relatively early stage. Additionally, c1, a relatively large (10,407 cells) mesenchymal subpopulation may also be involved in the mesenchymal to VSMC transition, particularly at a relatively late stage, as reflected by an abundance of potentially intermediate cells connecting.c1 and c3, mainly at stage ps51 (Figure 3D).

Altogether, our data suggest convergent development of the VSMCs at the base of the great arteries, where intermediate cell subpopulations were found to be involved in either mesenchymal to VSMC transition or myocardial to VSMC trans-differentiation. The inferred development paths of VSMCs are summarized in Figure 3F. Since a considerable number of intermediate cells were captured in our dataset, these biological processes could even be directly inferred from the tSNE plot (Figure 3G).

\section{Characteristics of gene expression profiles for intermediate cell subpopulations}

\section{involved in the myocardial to VSMC trans-differentiation}

We next sought to confirm myocardial to VSMC trans-differentiation by examining the gene expression profiles of the intermediate cell subpopulations that we identified above, c9 and c4. Cell cluster c9 and the largest myocardial cluster c2 displayed distinct expression profiles (Figure 4A). Compared with c2, c9 showed significant up-regulation of VSMC marker genes, 
including contractile VSMC markers ${ }^{29}$ (e.g., Acta2, Tagln, Cald1 and Myl9) and synthetic VSMC markers $^{36}$ (e.g., Eln, Colla2 and Cxcl12; Figure 4A, B). Myocardium-specific genes, Tnncl and Tnnt $2^{25}$, were expressed at significantly higher levels in $\mathrm{c} 9$ than in $\mathrm{c} 2$, reflecting the myocardial identity of c9. However, some myocardial markers, such as $M y l 2$, were significantly down-regulated in c9. Moreover, the ratio of Myh6 to Myh7, an index reflecting the degree of maturation and functionality of cardiomyocytes ${ }^{37}$, was significantly lower in c9 (Wilcoxon rank sum test $\mathrm{P}$-value $<2.2 \mathrm{e}-16$; Figure $4 \mathrm{C}$ ), suggesting that c9 cells may exhibit a less "mature" phenotype and be undergoing phenotypic changes. The genes up-regulated in c9 versus c2 (Online Table III) were enriched for pathways such as smooth muscle contraction, elastic fiber formation and artery morphogenesis (Figure 4D).

We also observed distinct expression profiles between the two VSMC clusters, c3 and c4 (Figure 4E, Online Table IV). Genes related to the development and maturation of VSMCs, such as Fbln 5, Eln, Col3al and Collal, were significantly up-regulated in c3 versus c4 (Figure $4 \mathrm{E} \& 4 \mathrm{~F}$ ). The genes up-regulated in $\mathrm{c} 3$ were mainly enriched for ECM organization (Figure $4 \mathrm{G})$. These results support our inference that $\mathrm{c} 3$ represents a more mature state of VSMCs than c4 does. Compared with c3, c4 exhibited significantly higher expression of both myocardial markers (e.g., Tnncl and Tnnt2 ${ }^{25}$ ) and mesenchymal markers (e.g. Cthrcl and Sox $9^{19}$ ), reflecting its myocardial and mesenchymal heritage.

Altogether, the expression profile analysis supports our inference that c9 and c4 cells are in an intermediate state along the trajectory of myocardium to VSMC trans-differentiation.

\section{Pseudo-temporal ordering and gene regulatory network analysis uncover critical}

transcriptional regulators potentially governing cell state transitions during OFT

\section{development.}

To elucidate gene expression dynamics, especially the transcriptional regulators governing the convergent development of VSMCs, we reconstructed the development trajectories for the different paths (Figure 3G) through pseudo-temporal ordering of individual cells using CellRouter (See Methods). For the myocardial to VSMC trans-differentiation (c9-c4-c3), we identified genes that were significantly correlated with the trajectory (Online Table V) and observed the loss of myocardial marker expression and gain of VSMC marker expression during the progression of trans-differentiation (Figure 5A). The Notch signaling pathway positively regulates the specification, differentiation, and maturation of VSMCs ${ }^{38}$. Strikingly, we noted that the expression of genes in the Notch signaling pathway, including receptor (Notchl), ligand ( $\mathrm{Jagl}$ l) and downstream targets (Heyl, Hey2, Heyl and Pdgfrb), was positively correlated with the trajectory (Figure 5B). Furthermore, by gene regulatory network (GRN) analysis, we obtained a set of key regulators that were activated sequentially along the trajectory and potentially drove the process (Figure 5C). We identified Heyl, encoding a known downstream TF of Notch signaling, at the top of the up-regulated regulators, consistent with its 
known positive role in VSMC development ${ }^{38}$. Tbx20, encoding a TF known as a transcriptional repressor in the developing heart ${ }^{39}$, was at the top of the down-regulated regulators, implying its role in repressing VSMC lineage-specifying genes. Regulators that were activated early in the trajectory, such as Plagll and Naca, may play potential roles in lineage commitment.

For mesenchymal (the c8 Penk ${ }^{+}$subpopulation) to VSMC transition (c8-c4-c3), we identified genes that were significantly correlated with the trajectory (Online Table VI) and observed the loss of mesenchymal marker expression and gain of VSMC marker expression during the progression of the transition (Figure 5D). The expression of genes in the Notch signaling pathway was positively correlated with the trajectory (Figure 5E). Interestingly, we found Heyl and Tbx20 also ranked at the top of the key regulators (Figure 5F). Consistent with our knowledge, the positive regulator Mef $2 c$, a TF essential for VSMC development ${ }^{40}$, was activated relatively early in the reconstructed trajectory.

Similarly, for $\mathrm{c} 1$ mesenchymal to VSMC transition $\left(\mathrm{c} 1_{\mathrm{ps} 51}-\mathrm{c} 3\right)$, we observed the loss of mesenchymal marker expression and gain of VSMC marker expression during the progression of the transition (Online Figure VA, Online Table VII). The expression of genes in the Notch signaling pathway was positively correlated with the trajectory (Online Figure VB). By contrast, the top regulators were different from those in the $\mathrm{c} 8$ mesenchymal to VSMC transition (Online Figure VC).

Additionally, we reconstructed the trajectory and identified the gene expression dynamics for the EndoMT process between c5 endothelial cells and c1 mesenchymal cells (Online Table VIII, Online Figure VIA). We observed the loss of endocardial marker expression and gain of mesenchymal marker expression during the progression of the transition (Online Figure VIB). The expression of genes that have been implicated in EndoMT, particularly genes of the TGF $\beta$ signaling pathway ${ }^{41}$, were positively correlated with the trajectory (Online Figure VIC). Furthermore, we identified the critical transcriptional regulators potentially involved in the transition (Online Figure VID). Interestingly, Klf2, encoding a TF that may play a role in EMT during cardiac development ${ }^{42}$, was found to be ranked at the top of critical regulators. The predicted targets of Klf2 were mainly enriched for epithelial to mesenchymal transition (Online Figure VIE), and included 16 genes that have been implicated in EMT (Gene Ontology term: epithelial to mesenchymal transition; Online Figure VIF).

\section{Convergent development of the VSMCs at the base of the great arteries is confirmed}

\section{by single-molecule fluorescent in situ hybridization}

To experimentally confirm myocardial to VSMC trans-differentiation, we performed singlemolecule fluorescent in situ hybridization (smFISH) with probes for $M y h 7$ (myocardial marker), Cxcl12 (VSMC marker) and Bmp4 (the myocardial subpopulation c9 marker), which were selected based on our single-cell dataset (Figure 2D). Serial sections of the OFT at the middle 
stage ps49 from proximal to distal clearly showed myocardial to arterial phenotypic change in OFT walls (Figure 6A). Cells expressing high levels of Myh7 (green) in OFT walls gradually changed into cells expressing Cxcl12 (blue) over development. Remarkably, this change occurred faster on the aortic side than on the pulmonary arterial side. In addition to OFT walls, the expression of $\mathrm{Cxcl12}$ was specifically observed in a strip of cushion mesenchymal cells between the lumens of aorta and pulmonary artery, which developed into the aorticopulmonary septum, a smooth muscle structure that eventually forms the facing walls of the great arteries. These results illustrated that $\mathrm{Cxcl12}$ could serve as an early specific marker for the VSMC lineage of the OFT. In a single section, we could observe cells expressing myocardial marker $M y h 7$ co-expressed various levels of Bmp 4 and the VSMC marker gene Cxcl12, indicating a continuum of cell state transitions during myocardial to VSMC trans-differentiation (Figure 6B). The $M y h 7^{+} \mathrm{Cxcl1} 2^{\text {low }} \mathrm{Bmp} 4^{\text {high }}$ cells that we observed (right panel of Figure 6B) may correspond to the $\mathrm{c} 9$ myocardial subpopulation.

Next, we tried to validate the existence of the $\mathrm{c} 8 \mathrm{Penk^{+ }}$ mesenchymal subpopulation and confirm its role in mesenchymal to VSMC transition. Interestingly, serial sections of the OFT from proximal to distal showed that the expression of Penk could be observed only at the cushion mesenchyme where the fusion was occurring (the section S12, Figure 6C). We observed Penk ${ }^{+}$

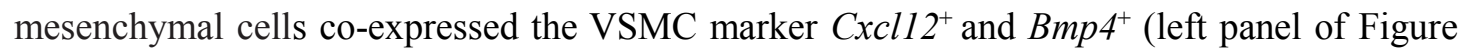
6D). These results suggest that the $\mathrm{c} 8 P e n k^{+}$mesenchymal subpopulation is undergoing transition to VSMCs and may be associated with the fusion of the OFT cushions. Additionally, at section S12, the co-expression of Bmp4 and Cxcl12 could be observed at both the mesenchyme of the aorticopulmonary septum (left panel of Figure 6D) and the myocardial free wall of the aortic side (right panel of Figure 6D), which imply that independent of the developmental paths, Bmp4 signaling may be associated with VSMC development.

\section{Web-based interfaces for further exploration of the single-cell data for the developing OFT}

Our dataset constitutes a valuable resource for the scientific community to prioritize the candidate genes of OFT malformations based on expression and map the candidate genes to cell types/subpopulations. To facilitate further data exploration, we developed web-based interfaces for our dataset (http://singlecelloft.fwgenetics.org). These tools permit interactive examination of expression for any gene of interest, dynamic changes in cell states for each cluster in a 3D space, and potential intercellular communications among cell lineages for any ligand-receptor pair. Based on the expression of known ligand-receptor pairs, we observed extensive networks of potential intercellular communications among all cell lineages at each developmental stage (Online Figure VII). Interestingly, the network became significantly denser at the middle stage ps49 than at the other two stages, indicating increased intercellular communications at the middle stage. 


\section{DISCUSSION}

In the present study, we performed single-cell transcriptomic sequencing of 55,611 mouse OFT cells from three successive developmental stages that generally correspond to the early, middle and late stages of OFT remodeling and septation (47, 49 and 51 pairs of somites). The largescale single-cell data empowered us to unbiasedly and systematically dissect the cellular diversity and heterogeneity during OFT development. We identified 17 cell clusters that could be assigned to six cell lineages. Among these lineages, the macrophage and VSMC lineages of the developing OFT have seldom been previously described in detail. In accordance with the myocardial to arterial phenotypic change, we observed the myocardial lineage diminished over time, while the VSMC lineage expanded during development. We provided molecular signatures for the cell lineages and clusters, and highlighted that $\mathrm{Cxcl1} 2$ could serve as a specific early marker for the embryonic VSMC lineage at the base of the great arteries. Cell lineage relationships and cellular transitions, such as EndoMT, have been identified through analyzing the dynamic changes in cell states by a force-directed layout of the KNN graph. In particular, we identified convergent development of the VSMCs at the base of the great arteries that has not been recognized before, where intermediate cell subpopulations were found to be involved in either myocardial to VSMC trans-differentiation or mesenchymal to VSMC transition. Through smFISH, we observed that cells expressing the myocardial marker $M y h 7$ co-expressed various levels of Bmp4 (the marker gene for the myocardial c9 cluster) and the VSMC marker gene Cxcl12 in OFT walls, thus confirming the existence of myocardial to VSMC transdifferentiation. Moreover, we found that the Penk ${ }^{+}$cluster c8, a relatively small mesenchymal subpopulation that was undergoing mesenchymal to VSMC transition, was specifically associated with the fusion of the OFT cushions. Through pseudo-temporal ordering and GRN analysis, we uncovered the expression dynamics and critical transcriptional regulators potentially governing cell state transitions during OFT development. Finally, we developed web-based interactive interfaces for our dataset to facilitate further data exploration.

\section{Cellular diversity of developing cardiac OFT uncovered by large-scale single-cell}

\section{profiling}

Defining the lineage, proportion and molecular signature of distinct cell types is fundamental to our understanding of developmental processes ${ }^{43}$. Single-cell RNA-seq has revolutionized developmental biology by allowing for unbiased and systematic characterization of the cellular states in developing systems, such as the developing human fetal kidney ${ }^{44}$ and prefrontal cortex $^{45}$. In a study on single-cell anatomical mapping of the embryonic heart ${ }^{19}$, the authors investigated the cellular composition and gene signatures of the OFT. However, only a total of 371 OFT cells (E10.5) were analyzed, which may be insufficient for a detailed dissection of heterogeneity. Apart from the four cell lineages (myocardial, epicardial, endocardial and mesenchymal) previously described ${ }^{19}$, our large-scale single-cell RNA-seq empowered us to detect a relatively rare $(0.5 \%)$ cell lineage, i.e., macrophages (Figure $2 \mathrm{~B}$ ). Notably, the relative 
proportion of macrophages did not significantly change during development (Figure 2C), implying their important role in OFT development. Given that apoptosis is a ubiquitous process during development including OFT development ${ }^{46}$, macrophages are thought to function to remove debris arising from normal apoptosis. Nevertheless, it has been increasingly recognized that macrophages residing in tissues play essential roles in normal development. For example, macrophages are required for coronary development via mediating the remodeling of the primitive coronary plexus ${ }^{47}$. Our findings highlight the role of macrophages in OFT remodeling and suggest avenues for further investigation into the role of macrophages in cardiac development.

We also characterized the VSMC lineage of the developing OFT which has not been described in previous studies ${ }^{19}$. Our data showed that the VSMC lineage constituted the second largest $(22.3 \%)$ cell population of the developing OFT (Figure 2B) and significantly expanded over development (Figure 2C). This observation is in line with our knowledge that OFT walls undergo myocardial to arterial phenotype change during development ${ }^{13}$. Although $\mathrm{Cxcl12}$ was previously found highly expressed in the walls of the aorta and pulmonary trunk of the embryonic heart (E12.5) ${ }^{28}$, our data highlights that $\mathrm{Cxcl12}$ could serve as a specific early marker for the embryonic VSMC lineage of the great arteries (Figure 2D). Through smFISH, the expression of $\mathrm{Cxcl} 12$ was observed specifically in cells that would eventually form mature smooth muscle structures. For example, Cxcl12 was highly expressed in a strip of cushion mesenchymal cells between the lumens of aorta and pulmonary artery, which would develop into the aorticopulmonary septum, a smooth muscle structure that eventually forms the facing walls of the great arteries (Figure 6A). The chemokine Cxcl12, which is secreted mainly in smooth muscle cells, has been suggested to be essential for coronary artery development through driving migration of cells expressing its receptor Cxcr4, e.g., endothelia cells ${ }^{28}$. Cxc112-Cxcr4 signaling has also been suggested to be required for correct patterning of pulmonary and aortic arch arteries possibly by protecting arteries from uncontrolled sprouting ${ }^{48}$. Although the mechanism underlying the arterial system development mediated by Cxc112Cxcr4 signaling remains elusive, our result may suggest a role in septation and remodeling of the OFT.

\section{Intra-lineage heterogeneity of developing cardiac OFT unraveled by large-scale}

\section{single-cell profiling}

Cellular heterogeneity is a general feature of biological tissues and exists even within seemingly 'homogeneous' cell populations ${ }^{16}$. The large-scale single-cell RNA-seq unraveled previously unrecognized cellular heterogeneity within each cell lineage of the developing OFT. Except for macrophages, all other five cell lineages displayed distinct cell clusters/subpopulations (Figure 1B, Figure 2B). Tmem 255a, previously reported to be an epicardial marker of the embryonic heart ${ }^{19}$, was found only mark one of the two subpopulations of the OFT epicardial lineage (Figure2D, Online Figure IV). Samples derived from each developmental stage were found to 
have cells in almost all of these clusters, and they differ only in terms of relative proportions (Figure 1C). This result illustrates that each of our samples captured a full spectrum of cellular states throughout development due to cellular asynchrony. This finding also gave us a unique chance to identify the intermediate, transitioning subpopulations that have not been characterized before. For example, we identified c9 as a myocardial subpopulation undergoing myocardium to VSMC trans-differentiation (Figure 4). Additionally, the transcriptomic heterogeneity among subpopulations was predominately driven by the cellular positions along the transition, differentiation or maturation of cell lineages, as reflected by the dynamic changes in relative proportions over time for many clusters (Figure 1C, Figure 3C). For example, the VSMC cluster $\mathrm{c} 3$ rapidly expanded over development and represented a more mature state of the VSMC lineage than $\mathrm{c} 4$ did. Nevertheless, transcriptomic heterogeneity may also be influenced by other factors. For example, c8 represents a small mesenchymal subpopulation associated with the fusion of OFT cushions.

\section{Myocardial to VSMC trans-differentiation occurred during the OFT development}

From the onset of development, the OFT is encased by a myocardial wall. As the progression of septation and remodeling, the myocardial wall rapidly changed into an arterial phenotype, characterized by the trick layer of smooth muscle cells in the tunica media. This myocardial to arterial phenotypic change has been previously described ${ }^{13,49}$, and our smFISH with probes marking the myocardial (Myh7) and VSMC (Cxcl12) lineages on serial sections of the OFT clearly displayed this process (Figure 6A). However, the fate of the myocardium during this process remains controversial to date. It has been suggested that trans-differentiation of myocardial cells to arterial components may occur during OFT development in the embryonic hearts of chicken ${ }^{50}$, rat $^{49}$ and mouse ${ }^{51}$. Conversely, it is also hold that the phenotypic change could just be considered a regression of the myocardium ${ }^{13}$. Our single-cell dataset supports the view of myocardial to VSMC trans-differentiation by identifying cell clusters representing a continuum of cell state transitions (Figure 3D). Expression profile comparison analysis demonstrated that myocardial cluster $\mathrm{c} 9$ and VSMC cluster $\mathrm{c} 4$ were in an intermediate state along the trajectory of myocardium to VSMC trans-differentiation (Figure 4). Through smFISH we observed that cells expressing myocardial marker $M y h 7$ co-expressed various levels of Bmp4 (c9 marker gene) and VSMC marker gene Cxcl12 in OFT walls, thus confirming the myocardial to VSMC trans-differentiation (Figure 6). A recent study demonstrated that myocardial cells may transit to the mesenchymal cells of the intercalated cushions during OFT development ${ }^{52}$. Thus, our findings provide additional evidence that highlights the plasticity of the embryonic myocardial cells of the OFT. All these findings imply that transitions between cell lineages during OFT development may be more complicated than previously appreciated.

\section{Convergent development of the VSMCs at the base of the great arteries}

Cell lineage relationships and cellular state transitions can be inferred from time-series single- 
cell transcriptomic data even for a complex developmental system, such as embryogenesis of frog $^{53}$ and zebrafish ${ }^{54}$, through a force-directed layout of the KNN graph. Based on the dynamic change in cell states over time reflected by the KNN graph of our time-series single-cell data, known cell transition events were recapitulated, for example, EndoMT that underwent between the endocardial and mesenchymal subpopulations (Online Figure VIA). In particular, we identified convergent development of the VSMCs at the base of the great arteries that has not been recognized before, where intermediate cell subpopulations were found to be involved in either myocardial to VSMC trans-differentiation or mesenchymal to VSMC transition (Figure 3F \&3G). We found that the mesenchymal to VSMC transition involved one mesenchymal subpopulation, c1, that occurred mainly at the late stage, and another smaller mesenchymal subpopulation, c8, that occurred mainly at the early stage. Such a temporal relationship for mesenchymal to VSMC transition has never been recognized before. Furthermore, by smFISH, the c8 Penk ${ }^{+}$subpopulation was found to be specifically associated with the fusion of OFT cushions, in line with its relatively small size and populating the early stage samples (Figure $6 \mathrm{C}$ ). In addition to the mesenchymal subpopulations, the myocardial subpopulation c9 also contributed to the development of the VSMC lineage. Altogether, three developmental paths were identified to be implicated in convergent development of VSMC lineage at the base of the great arteries, which involves different cell lineages and different subpopulations of the same lineage.

Furthermore, by pseudo-temporal ordering and GRN analysis, we uncovered gene expression dynamics and critical transcriptional regulators potentially governing the cell state transitions during the development of VSMCs (Figure 5, Online Figure V). The Notch signaling pathway has been known to positively regulate the specification, differentiation, and maturation of $\mathrm{VSMCs}^{38}$. We found that the expression of genes in the Notch signaling pathway, including receptor (Notchl), ligand (Jagl) and downstream targets (Heyl, Hey2, Heyl and Pdgfrb), was positively correlated with the reconstructed trajectories for all the three paths. Then, we identified Heyl, encoding a known downstream TF of the Notch signaling, at the top of the upregulated regulators. Thus, our results highlight the role of the Notch signaling pathway in the development of the OFT VSMC lineage. We provide a set of critical transcriptional regulators that were sequentially activated or repressed along the development trajectory. For many of these regulators, the roles in VSMC development have seldom been suggested before.

In conclusion, through large-scale single-cell transcriptomic sequencing, we performed an unbiased and systematic study on the cellular types and states of the cardiac OFT during development. Our results support the existence of myocardial to VSMC trans-differentiation, and convergent development of the VSMC lineage at the base of the great arteries. We provide a single-cell reference map of cell states for normal OFT development, which allows the CHD community to assess how perturbations affect the transcriptomic states of OFT cell lineages, to prioritize candidate genes of OFT malformations based on expression, and to map candidate genes to cell types or subpopulations. Our study demonstrated the power of time-series singlecell transcriptomic data for identifying cell state transitions in a complex developmental system. 
We thank the staff in the public platform of the State Key Laboratory of Cardiovascular Disease for providing technical assistance.

\section{SOURCES OF FUNDING}

489 This work is supported by grants from the CAMS Initiative for Innovative Medicine (2016490 I2M-1-016) and the Post-doctoral International Exchange Project of Peking Union Medical 491 College.

\section{DISCLOSURES}

None

494

495

496

497

498

499

500

501

502

503

504

505

506

507

508

509

510

511

\section{REFERENCES}

1. van der Linde D, Konings EE, Slager MA, Witsenburg M, Helbing WA, Takkenberg JJ and RoosHesselink JW. Birth prevalence of congenital heart disease worldwide: a systematic review and metaanalysis. J Am Coll Cardiol. 2011;58:2241-2247.

2. Thom T, Haase N, Rosamond W, Howard VJ, Rumsfeld J, Manolio T, Zheng Z-J, Flegal K, O'donnell C and Kittner S. Heart disease and stroke statistics - 2006 update: a report from the American Heart Association Statistics Committee and Stroke Statistics Subcommittee. Circulation. 2006;113:e85e151.

3. Neeb Z, Lajiness JD, Bolanis E and Conway SJ. Cardiac outflow tract anomalies. Wiley Interdiscip Rev Dev Biol. 2013;2:499-530.

4. Kelly RG, Buckingham ME and Moorman AF. Heart fields and cardiac morphogenesis. Cold Spring Harb Perspect Med. 2014;4.

5. Erikssen G, Liestøl K, Seem E, Birkeland S, Saatvedt KJ, Hoel TN, Døhlen G, Skulstad H, Svennevig JL and Thaulow E. Achievements in congenital heart defect surgery: a prospective, 40-year study of 7038 patients. Circulation. 2015;131:337-346.

6. Webb S, Qayyum SR, Anderson RH, Lamers WH and K Richardson M. Septation and separation within the outflow tract of the developing heart. J Anat. 2003;202:327-342.

7. Verzi MP, McCulley DJ, De Val S, Dodou E and Black BL. The right ventricle, outflow tract, and ventricular septum comprise a restricted expression domain within the secondary/anterior heart field. Dev Biol. 2005;287:134-145.

8. Eisenberg LM and Markwald RR. Molecular regulation of atrioventricular valvuloseptal morphogenesis. Circ Res. 1995;77:1-6.

9. Plein A, Fantin A and Ruhrberg C. Neural crest cells in cardiovascular development. Curr Top Dev Biol. 2015;111:183-200.

10. Lin CJ, Lin CY, Chen CH, Zhou B and Chang CP. Partitioning the heart: mechanisms of cardiac septation and valve development. Development. 2012;139:3277-3299.

11. Kelly RG and Buckingham ME. The anterior heart-forming field: voyage to the arterial pole of the 
heart. Trends Genet. 2002;18:210-216.

522 12. von Gise A and Pu WT. Endocardial and epicardial epithelial to mesenchymal transitions in heart development and disease. Circ Res. 2012;110:1628-45. formation of the ventricular outflow tracts, arterial valves, and intrapericardial arterial trunks. Heart. 2003;89:1110-8.

14. Zheng GX, Terry JM, Belgrader P, Ryvkin P, Bent ZW, Wilson R, Ziraldo SB, Wheeler TD, McDermott GP, Zhu J, Gregory MT, Shuga J, Montesclaros L, Underwood JG, Masquelier DA, Nishimura SY, Schnall-Levin M, Wyatt PW, Hindson CM, Bharadwaj R, Wong A, Ness KD, Beppu LW, Deeg HJ, McFarland C, Loeb KR, Valente WJ, Ericson NG, Stevens EA, Radich JP, Mikkelsen TS, Hindson BJ and Bielas JH. Massively parallel digital transcriptional profiling of single cells. Nat Commun. 2017;8:14049.

15. Potter SS. Single-cell RNA sequencing for the study of development, physiology and disease. Nat Rev Nephrol. 2018;14:479-492.

16. Wen L and Tang F. Single-cell sequencing in stem cell biology. Genome Biol. 2016;17:71.

17. Herring CA, Chen B, McKinley ET and Lau KS. Single-Cell Computational Strategies for Lineage Reconstruction in Tissue Systems. Cell Mol Gastroenterol Hepatol. 2018;5:539-548. J, Gray J, Pu W, Bruneau BG, Seidman JG and Seidman CE. Single-Cell Resolution of Temporal Gene Expression during Heart Development. Dev Cell. 2016;39:480-490. SM. Transcriptomic Profiling Maps Anatomically Patterned Subpopulations among Single Embryonic Cardiac Cells. Dev Cell. 2016;39:491-507. K, Rosenthal NA and Tallquist MD. Revisiting Cardiac Cellular Composition. Circ Res. 2016;118:4009. Single-cell transcriptional profiling reveals cellular diversity and intercommunication in the mouse Heart. Cell Rep. 2018;22:600-610. Gordonov S, Mazloom AR, Ma'ayan A, Chua WJ, Hansen TH, Turley SJ, Merad M, Randolph GJ and Immunological Genome C. Gene-expression profiles and transcriptional regulatory pathways that underlie the identity and diversity of mouse tissue macrophages. Nat Immunol. 2012;13:1118-28. $\mathrm{K}$ and Kispert A. Upk3b is dispensable for development and integrity of urothelium and mesothelium. PLOS ONE. 2014;9:e112112.

557 24. Narumiya H, Hidaka K, Shirai M, Terami H, Aburatani H and Morisaki T. Endocardiogenesis in 558 embryoid bodies: novel markers identified by gene expression profiling. Biochem Biophys Res Commun. $559 \quad 2007 ; 357: 896-902$.

560 25. Wilczewski CM, Hepperla AJ, Shimbo T, Wasson L, Robbe ZL, Davis IJ, Wade PA and Conlon FL. 561 CHD4 and the NuRD complex directly control cardiac sarcomere formation. Proc Natl Acad Sci U S A. $562 \quad 2018 ; 115: 6727-6732$.

563 26. Gunaje JJ, Bahrami AJ, Schwartz SM, Daum G and Mahoney Jr WM. PDGF-dependent regulation 
of regulator of $\mathrm{G}$ protein signaling-5 expression and vascular smooth muscle cell functionality. $A m \mathrm{~J}$ Physiol Cell Physiol. 2011;301:C478-C489.

27. Daniel J-M, Prock A, Dutzmann J, Sonnenschein K, Thum T, Bauersachs J and Sedding DG. Regulator of G-protein signaling 5 prevents smooth muscle cell proliferation and attenuates neointima formation. Arterioscler Thromb Vasc Biol. 2016;36:317-327.

28. Ivins S, Chappell J, Vernay B, Suntharalingham J, Martineau A, Mohun TJ and Scambler PJ. The CXCL12/CXCR4 axis plays a critical role in coronary artery development. Dev Cell. 2015;33:455-468. 29. Sinha S, Iyer D and Granata A. Embryonic origins of human vascular smooth muscle cells: implications for in vitro modeling and clinical application. Cell Mol Life Sci. 2014;71:2271-88.

30. Rudat C and Kispert A. Wt1 and epicardial fate mapping. Circ Res. 2012;111:165-169.

31. Henderson DJ and Copp AJ. Versican expression is associated with chamber specification, septation, and valvulogenesis in the developing mouse heart. Circ Res. 1998;83:523-532.

32. Mahdavi V, Periasamy M and Nadal-Ginard B. Molecular characterization of two myosin heavy chain genes expressed in the adult heart. Nature. 1982;297:659.

33. Xu K, Chong DC, Rankin SA, Zorn AM and Cleaver O. Rasip1 is required for endothelial cell motility, angiogenesis and vessel formation. Dev Biol. 2009;329:269-279.

34. Spencer JA, Hacker SL, Davis EC, Mecham RP, Knutsen RH, Li DY, Gerard RD, Richardson JA, Olson EN and Yanagisawa H. Altered vascular remodeling in fibulin-5-deficient mice reveals a role of fibulin-5 in smooth muscle cell proliferation and migration. Proc Natl Acad Sci U S A. 2005;102:29462951.

35. Weinreb C, Wolock S, Klein AM and Berger B. SPRING: a kinetic interface for visualizing high dimensional single-cell expression data. Bioinformatics. 2018;34:1246-1248.

36. Xu J and Shi G-P. Vascular wall extracellular matrix proteins and vascular diseases. Biochim Biophys Acta Mol Basis Dis. 2014;1842:2106-2119.

37. Pandya $\mathrm{K}$ and Smithies O. $\beta-\mathrm{MyHC}$ and cardiac hypertrophy: size does matter. Circ Res. 2011;109:609-610.

38. Fouillade C, Monet-Lepretre M, Baron-Menguy C and Joutel A. Notch signalling in smooth muscle cells during development and disease. Cardiovasc Res. 2012;95:138-46.

39. Singh R and Kispert A. Tbx20, Smads, and the atrioventricular canal. Trends Cardiovasc Med. 2010;20:109-114.

40. Lin Q, Lu J, Yanagisawa H, Webb R, Lyons GE, Richardson JA and Olson EN. Requirement of the MADS-box transcription factor MEF2C for vascular development. Development. 1998;125:4565-4574. 41. Cho JG, Lee A, Chang W, Lee M-S and Kim J. Endothelial to mesenchymal transition represents a key link in the interaction between inflammation and endothelial dysfunction. Front Immunol. 2018;9. 42. Chiplunkar AR, Lung TK, Alhashem Y, Koppenhaver BA, Salloum FN, Kukreja RC, Haar JL and Lloyd JA. Krüppel-like factor 2 is required for normal mouse cardiac development. PLOS ONE. 2013;8:e54891.

43. Griffiths JA, Scialdone A and Marioni JC. Using single-cell genomics to understand developmental processes and cell fate decisions. Mol Syst Biol. 2018;14:e8046.

44. Wang P, Chen Y, Yong J, Cui Y, Wang R, Wen L, Qiao J and Tang F. Dissecting the global dynamic molecular profiles of human fetal kidney development by single-cell RNA sequencing. Cell Rep. 2018;24:3554-3567.e3.

45. Zhong S, Zhang S, Fan X, Wu Q, Yan L, Dong J, Zhang H, Li L, Sun L, Pan N, Xu X, Tang F, 
607 Zhang J, Qiao J and Wang X. A single-cell RNA-seq survey of the developmental landscape of the human

608 prefrontal cortex. Nature. 2018;555:524-528.

609 46. Fisher SA, Langille BL and Srivastava D. Apoptosis during cardiovascular development. Circ Res. $610 \quad 2000 ; 87: 856-864$.

611 47. Leid J, Carrelha J, Boukarabila H, Epelman S, Jacobsen SEW and Lavine KJ. Primitive embryonic 612 macrophages are required for coronary development and maturation. Circ Res. 2016;118:1498-1511.

613 48. Kim BG, Kim YH, Stanley EL, Garrido-Martin EM, Lee YJ and Oh SP. CXCL12-CXCR4 614 signalling plays an essential role in proper patterning of aortic arch and pulmonary arteries. Cardiovasc 615 Res. 2017;113:1677-1687.

616 49. Ya J, van den Hoff MJ, de Boer PA, Tesink-Taekema S, Franco D, Moorman AF and Lamers WH. 617 Normal development of the outflow tract in the rat. Circ Res. 1998;82:464-72.

618 50. Argüello C, Mv DLC and Sánchez C. Ultrastructural and experimental evidence of myocardial cell 619 differentiation into connective tissue cells in embryonic chick heart. J Mol Cell Cardiol. 1978;10:307620315.

621 51. Yang YP, Li HR and Jing Y. Septation and shortening of outflow tract in embryonic mouse heart 622 involve changes in cardiomyocyte phenotype and $\alpha$-SMA positive cells in the endocardium. Chin Med J 623 (Engl). 2004;117:1240-1245.

624 52. Mifflin JJ, Dupuis LE, Alcala NE, Russell LG and Kern CB. Intercalated cushion cells within the 625 cardiac outflow tract are derived from the myocardial troponin T type 2 (Tnnt2) Cre lineage. Dev Dyn. $6262018 ; 247: 1005-1017$.

627 53. Briggs JA, Weinreb C, Wagner DE, Megason S, Peshkin L, Kirschner MW and Klein AM. The 628 dynamics of gene expression in vertebrate embryogenesis at single-cell resolution. Science. 629 2018;360:eaar5780.

630 54. Wagner DE, Weinreb C, Collins ZM, Briggs JA, Megason SG and Klein AM. Single-cell mapping 631 of gene expression landscapes and lineage in the zebrafish embryo. Science. 2018;360:pp. 981-987. 
Figure 1. Single-cell transcriptomic sequencing and unbiased clustering of cells during three successive stages of mouse OFT development were captured and sequenced separately. Two biological replicates were prepared for each stage. ps47, ps49 and ps51 denote 47, 49 and 51 pairs of somites, respectively. Dissection boundaries are indicated by the red dotted lines on the schematic plots of embryonic hearts. (B) Unsupervised clustering of all cells reveals 17 cell clusters projected on a two-dimensional tSNE map. (C) Fraction of cells derived from each sample for each cluster. All samples are normalized to the same number of cells $(4,270)$.

Figure 2. Cellular diversity and gene signatures of the developing OFT identified by single-cell transcriptomic analysis. (A) Cell lineages recognized by known cell-type specific marker genes. Each cell is colored according to the scaled expression of the indicated marker gene. (B) Relatedness of clusters revealed by hierarchical clustering. This analysis is based on the average expression of the 1,381 HVGs in each cluster. (C) Cell fractions of each stage for each cell lineage. The average cell fraction of the two biological replicates and the standard error are shown on the bar plot. 'ns': not significant; *: Student's t-Test P-value $<0.05$; **: Student's t-Test $\mathrm{P}$-value $<0.01$. In $\mathrm{B}$ and $\mathrm{C}$, all samples are normalized to the same number of cells $(4,270)$. (D) Gene signature for each cell lineage or cluster. These genes were identified and selected by differential expression analysis and random forest classification. The schematic plot represents a cross section of OFT in a specific position where the aorta wall shows an artery phenotype while the wall of the pulmonary artery still possesses a myocardial phenotype. The two small myocardial clusters $\mathrm{c} 14$ and $\mathrm{c} 16$ were not incorporated in this analysis. EP: epicardial; MS: mesenchymal; MC: myocardial; ED: endocardial; VSMC: vascular smooth muscle cell

Figure 3. Convergent development of the VSMCs at the base of the great arteries. (A) Force-directed layout of a KNN graph showing a continuous expression topology of the OFT cellulome during development. Each dot denotes a cell colored by cell cluster. (B) KNN graph colored by development stage. (C) Dynamic changes of cell states over time. Cells colored in gray denote those from other stages. All samples are normalized to the same number of cells $(4,270)$. (D) Dynamic changes of cell clusters directly involved in VSMC development. (E) Relative proportions of cell clusters directly involved in VSMC development. (F) Inferred developmental paths of the smooth muscle cells displayed on the KNN graph. (G) Inferred developmental paths of the smooth muscle cells displayed on the tSNE plot. 
665

666

667

668

669

670

671

672

673

674

675

676

677

678

679

680

681

682

683

684

685

686

687

688

689

690

691

692

693

694

695

696

697

Figure 4. Characteristics of gene expression profiles for the intermediate cell subpopulations during myocardial to VSMC trans-differentiation. (A) Heatmap showing the DEGs between myocardial cluster c2 and c9. (B) Significant expression differences of some key marker genes between c2 and c9. Each dot denotes a cell. (C) The ratio of Myh6 to Myh7 in c2 and c9. (D) Functional enrichment of genes significantly up-regulated in c9. (E) Heatmap showing the DEGs between the VSMC cluster $\mathrm{c} 3$ and the intermediate VSMC population $\mathrm{c} 4$. (F) Significant expression differences of some key marker genes between c3 and c4. (G) Functional enrichment of genes significantly up-regulated in c3.

Figure 5. Pseudo-temporal ordering and GRN analysis uncover critical transcriptional regulators potentially governing cell state transitions during the development of VSMCs. (A) Loss of myocardial marker expression and gain of VSMC marker expression during myocardial to VSMC trans-differentiation. (B) Expression of genes in the Notch signaling is positively correlated with the trajectory of myocardial to VSMC trans-differentiation. (C) Critical transcriptional regulators potentially involved in myocardial to VSMC transdifferentiation. (D) Loss of mesenchymal marker expression and gain of VSMC marker expression during c8 mesenchymal to VSMC transition. (E) Expression of genes in the Notch signaling is positively correlated with the trajectory of $\mathrm{c} 8$ mesenchymal to VSMC transition. (F) Critical transcriptional regulators potentially involved in c8 mesenchymal to VSMC transition. $\bullet$ : VSMC marker; $\boldsymbol{\Delta}$ : myocardial marker; $\star$ : mesenchymal marker.

Figure 6. Convergent development of the VSMCs at the base of the great arteries is confirmed by smFISH. (A) Serial sections of the OFT at stage ps49 from proximal to distal showing myocardial to arterial phenotypic change in the OFT walls. Green: Myh7; Blue: Cxcl12; Red: Bmp4. Scale bar: $50 \mu \mathrm{m}$. The aorta is arranged on the upper side. (B) Myocardial to VSMC trans-differentiation is supported by the observation that cells expressing myocardial marker $M y h 7$ co-express various levels of Bmp 4 and VSMC marker gene Cxcl12. Middle panel: section S18; The yellow dotted line shows the border of the lumen of aorta (A) and pulmonary trunk (P); AS: aorticopulmonary septum. Left panel: $M y h 7^{+} C x c l 12^{\text {high }} B m p 4^{\text {low }}$ cells; Right panel: $M y h 7^{+} \mathrm{Cxcl1} 2^{\text {low }} \mathrm{Bmp} 4^{\text {high }}$ cells. (C) Serial sections of the OFT at the stage ps49 from proximal to distal showing that the $\mathrm{c} 8 \mathrm{Penk}^{+}$mesenchymal subpopulation is undergoing transition to VSMCs and is associated with the fusion of the OFT cushions. Green: Penk; Blue: Cxcl1 2; Red: Bmp4. Scale bar: $50 \mu \mathrm{m}$. (D) Co-expression of Penk, Cxcl12 and Bmp4. The arrow indicates the location where the fusion is occurring. Middle panel: section S12; Left panel:

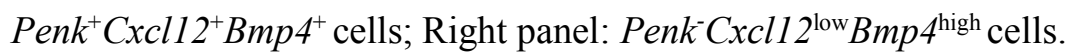


bioRxiv preprint doi: https://doi.org/10.1101/469346; this version posted November 13, 2018. The copyright holder for this preprint (which was not certified by peer review) is the author/funder, who has granted bioRxiv a license to display the preprint in perpetuity. It is made available under aCC-BY-NC-ND 4.0 International license.

A

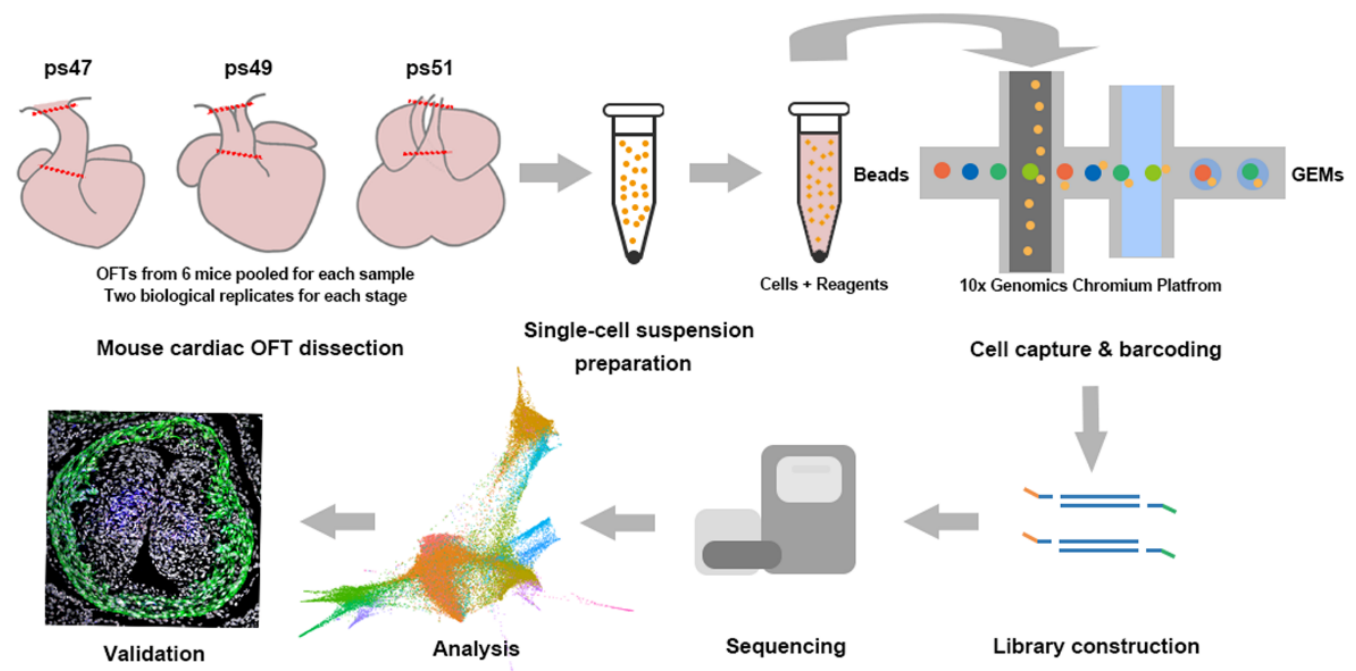

B

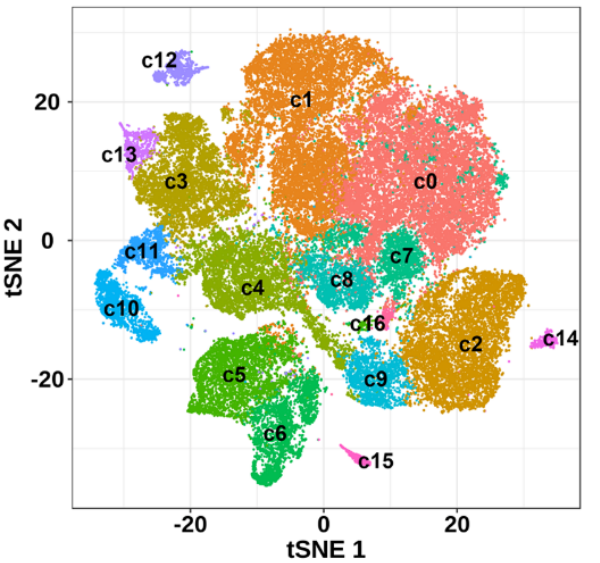

tSNE 1
C $\quad$ ps47_1 $1 \square$ ps47_2 $\square$ ps49_1 $\square$ ps49_2 $\square$ ps51_1 1 ps51_2

co Mesenchymal c1 Mesenchymal c2 Myocardia

c3 vSMc

C5 Endocarda

c6 Endocardial

c7 Mesenchymal

c8 Mesenchymal

c9 Myocardial

c10 Epicardial

c11 Epicardial

c12 Endocardial

c13 vsmc

c14 Myocardial

c15 Macrophage

c16 Myocardial

699

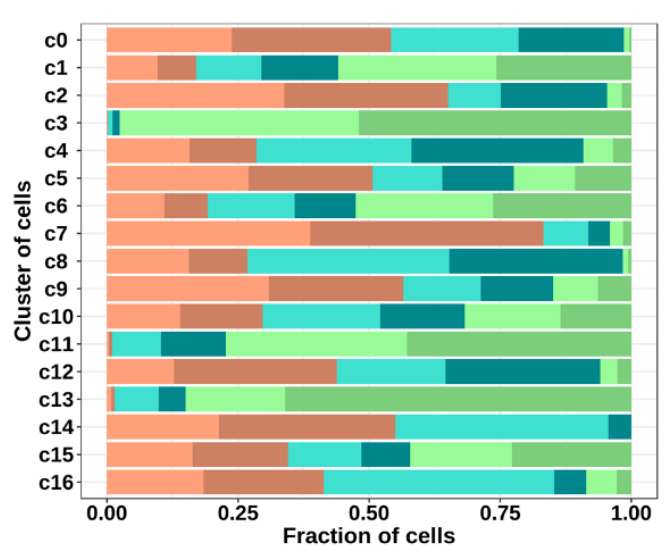

Figure 1 


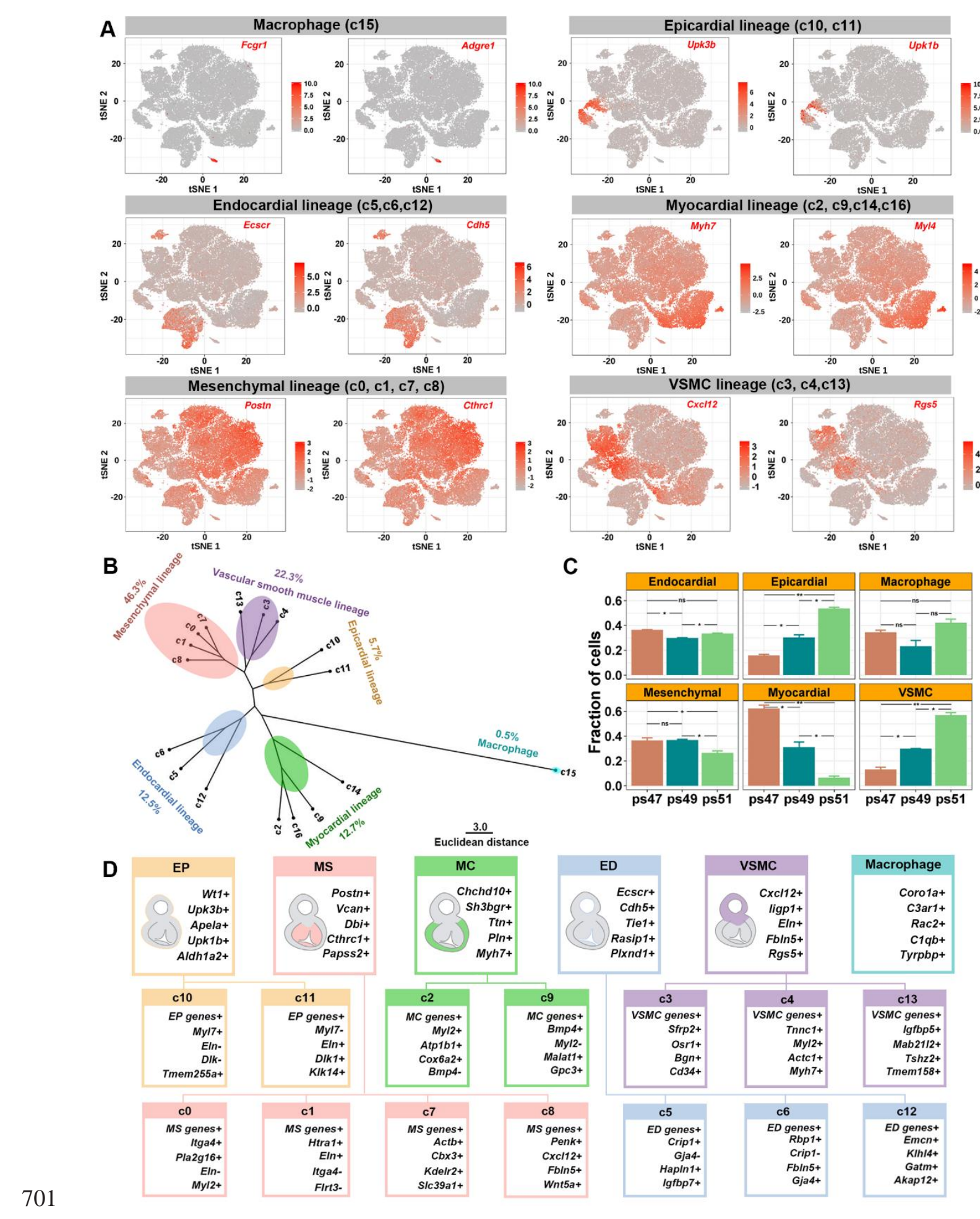


bioRxiv preprint doi: https://doi.org/10.1101/469346; this version posted November 13, 2018. The copyright holder for this preprint (which was not certified by peer review) is the author/funder, who has granted bioRxiv a license to display the preprint in perpetuity. It is made available under aCC-BY-NC-ND 4.0 International license.

A
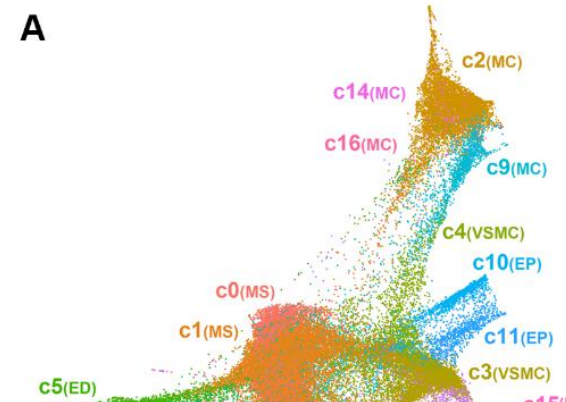

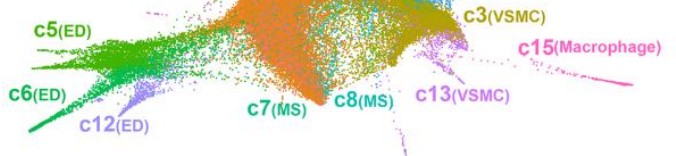

C

ps 47
$(8,540$ cells $)$
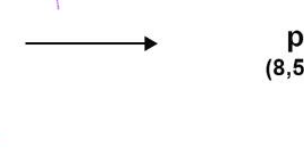

ps49

8,540 cells

B

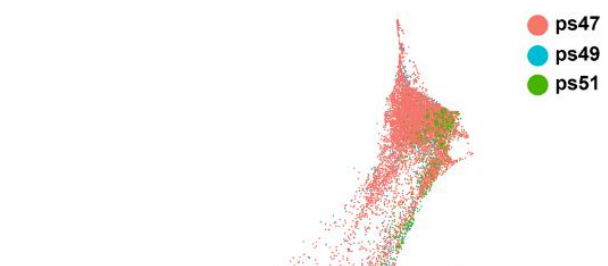

ps47
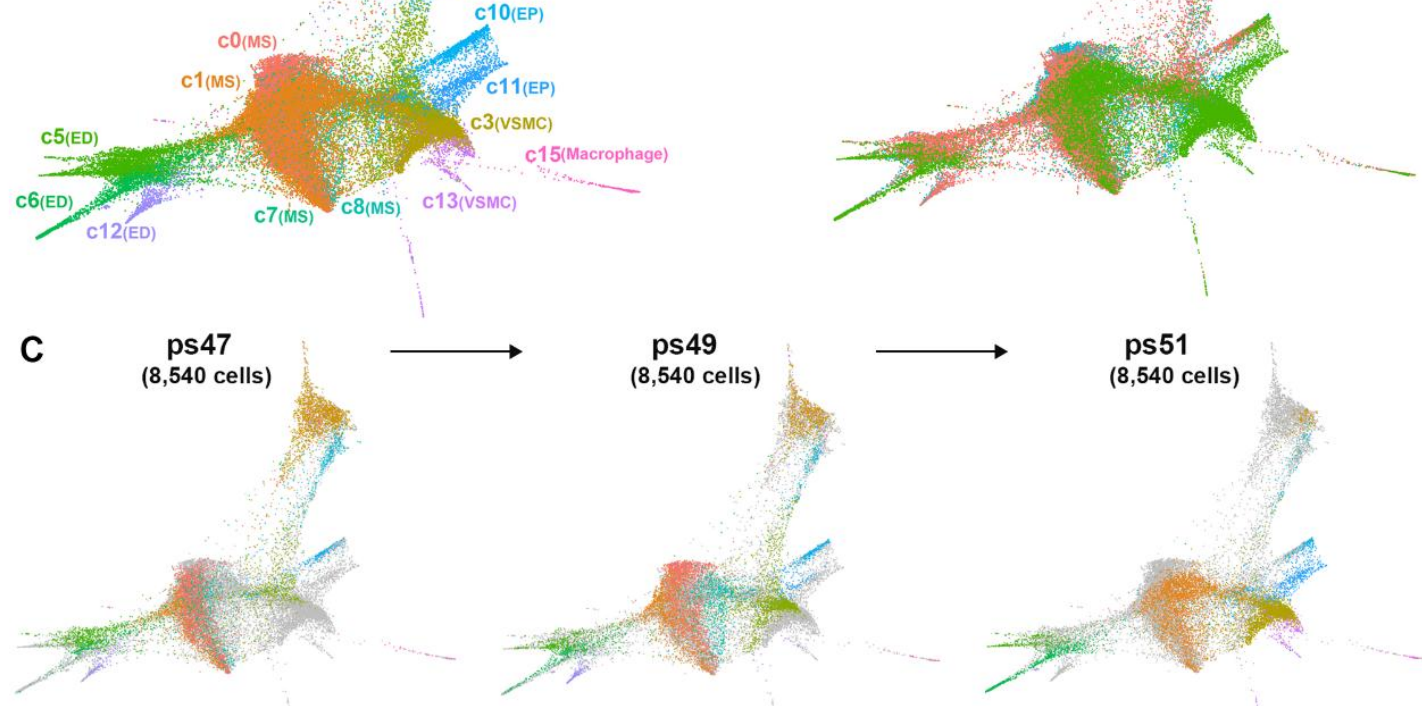

D

c1
c3
c4
c8
c9

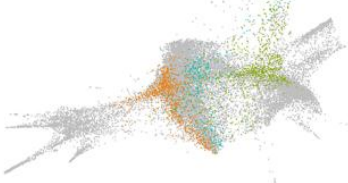

E

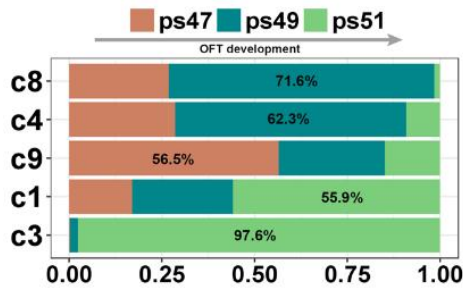

703
F
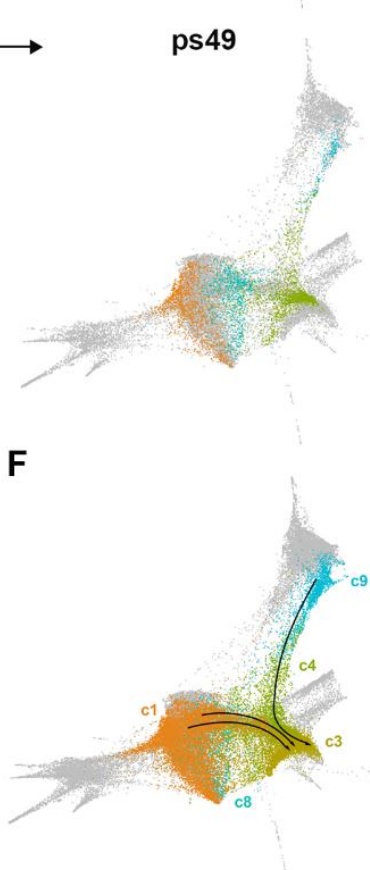

ps51

(8,540 cells)

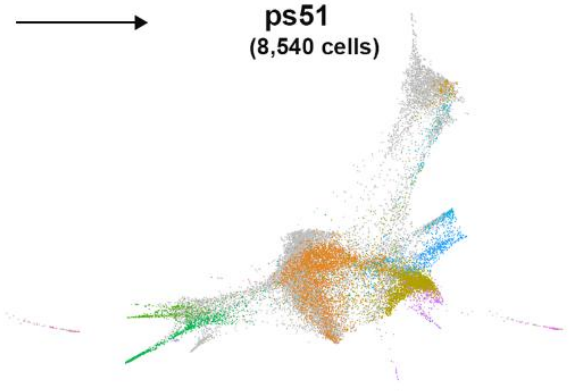

ps51

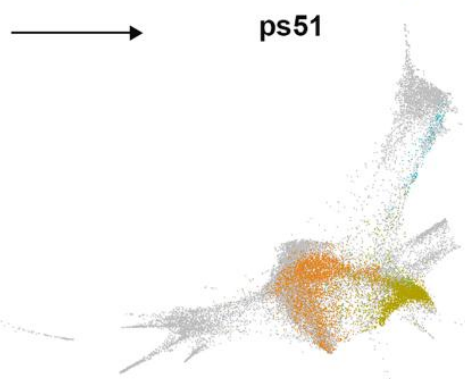

G

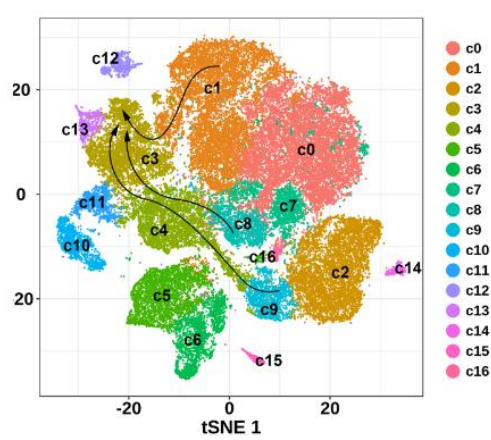


bioRxiv preprint doi: https://doi.org/10.1101/469346; this version posted November 13, 2018. The copyright holder for this preprint (which was not certified by peer review) is the author/funder, who has granted bioRxiv a license to display the preprint in perpetuity. It is made available under aCC-BY-NC-ND 4.0 International license.

A

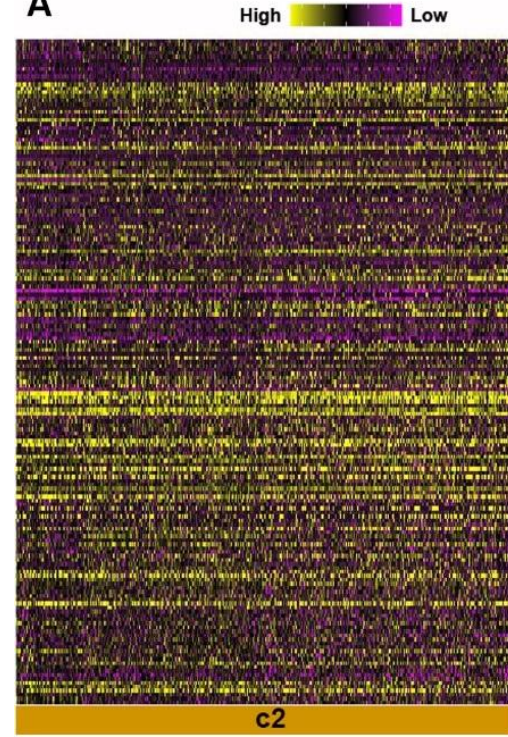

C

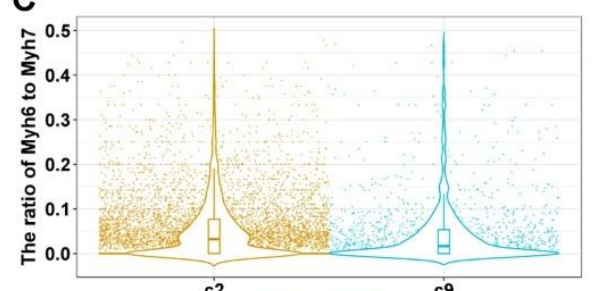

E

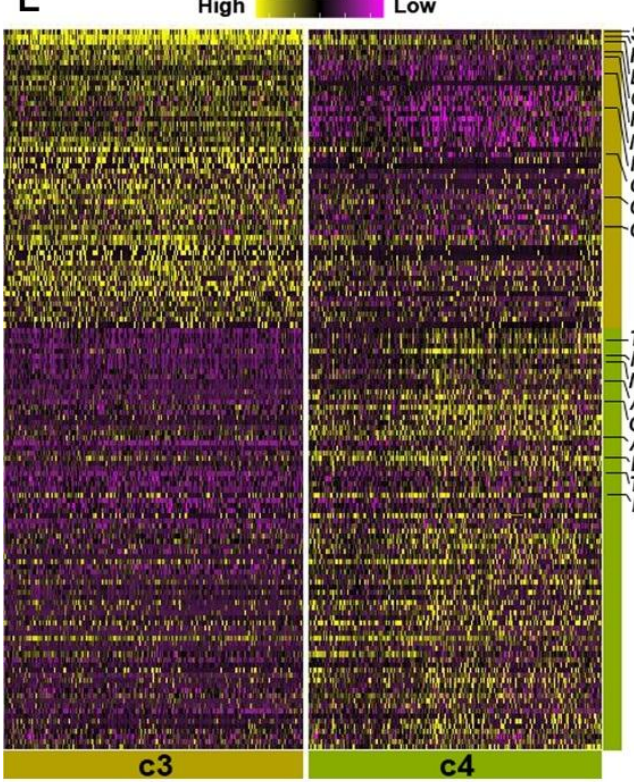

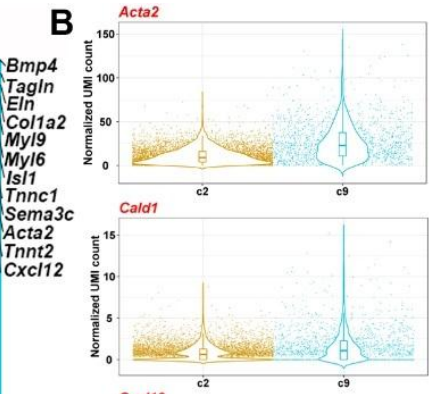
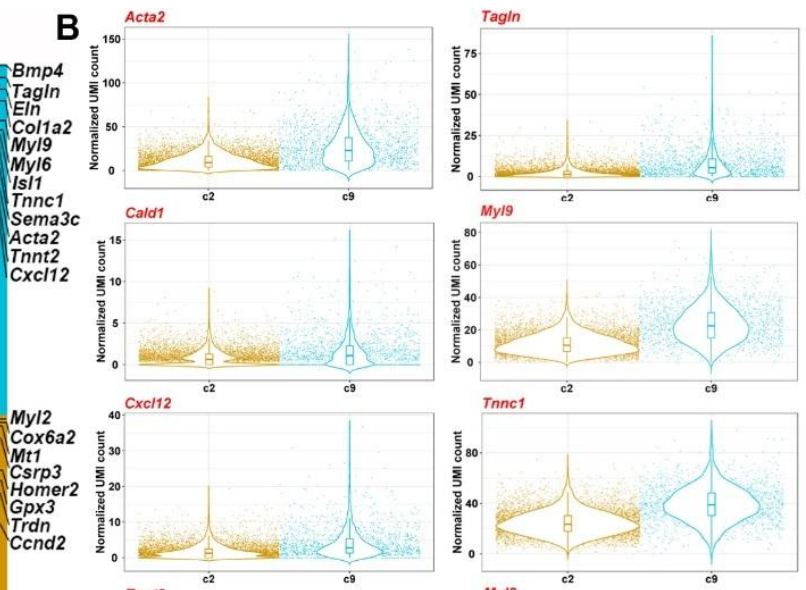

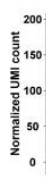

D

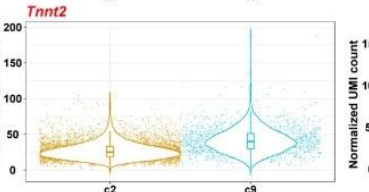

Myl2

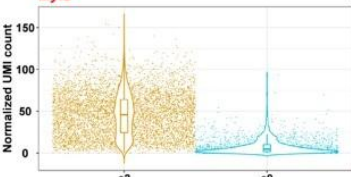

R-HSA-445355: smooth muscle contraction R-HSA-0003007: heart morphogenesis GO:0048738: cardiac muscle tissue development R-HSA-2129379: molecules associated with elastic fibre

GO:0003151: outflow tract morphogenesis :0008593: regulation of Notch signaling pathway GO:0003148: outflow tract septum morphogenesis GO:0048662: negative regulation of smooth muscle cell proliferation GO:0051149: positive regulation of muscle cell differentiation

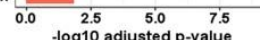
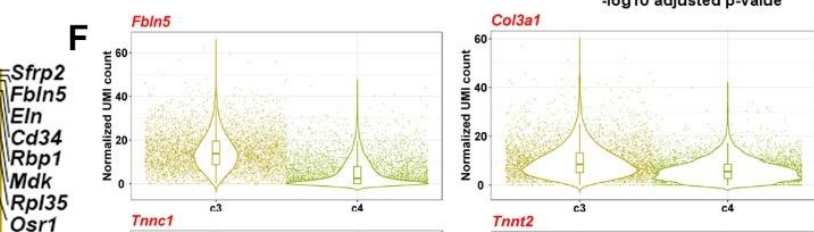

Osr1 Colla1

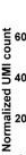

Tnnc1 Myl2
Myh7 Mnkrd1 Ankrd1 Apoe Tbx20 Is/1
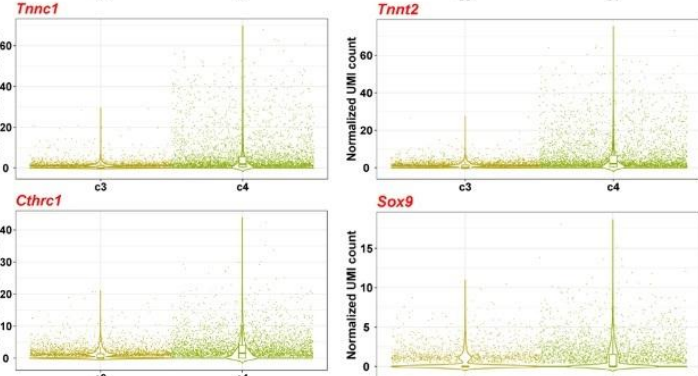

G

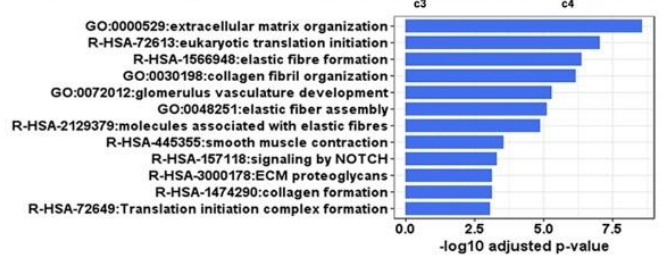



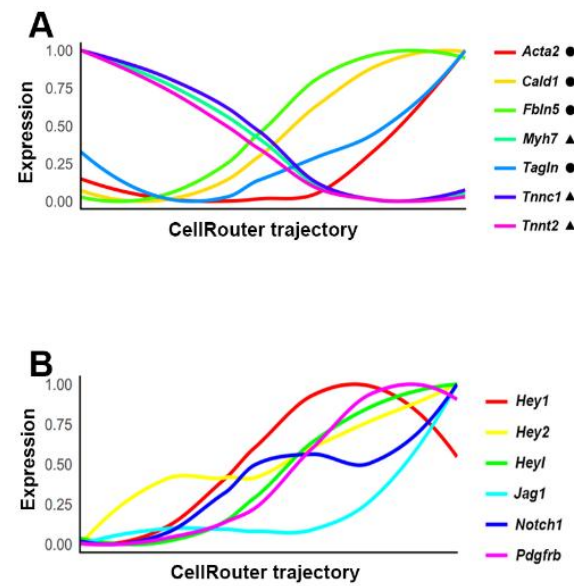

D
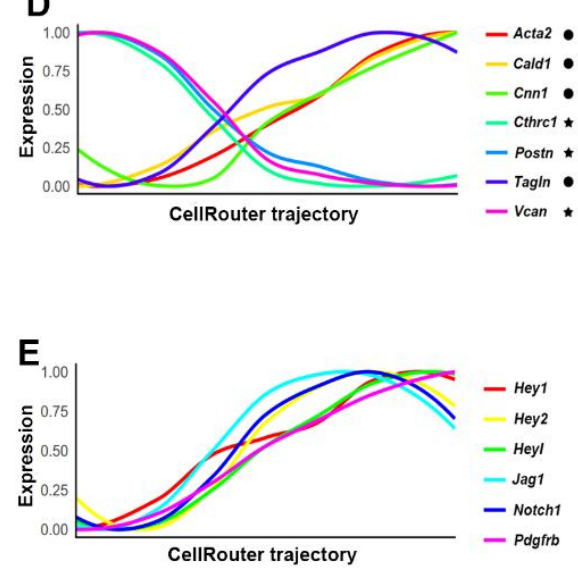

C
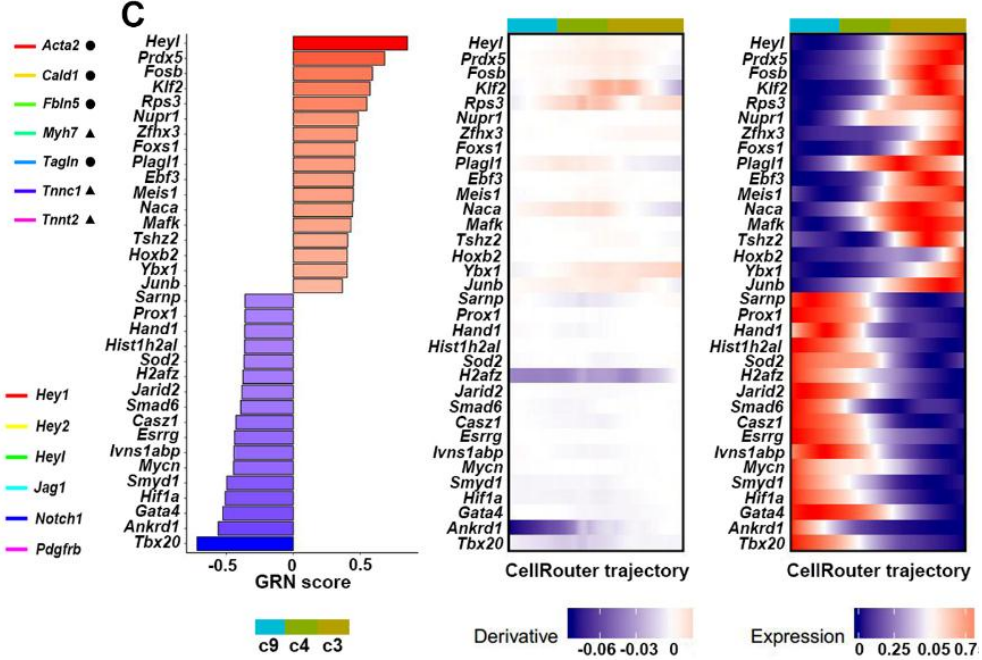

$F$
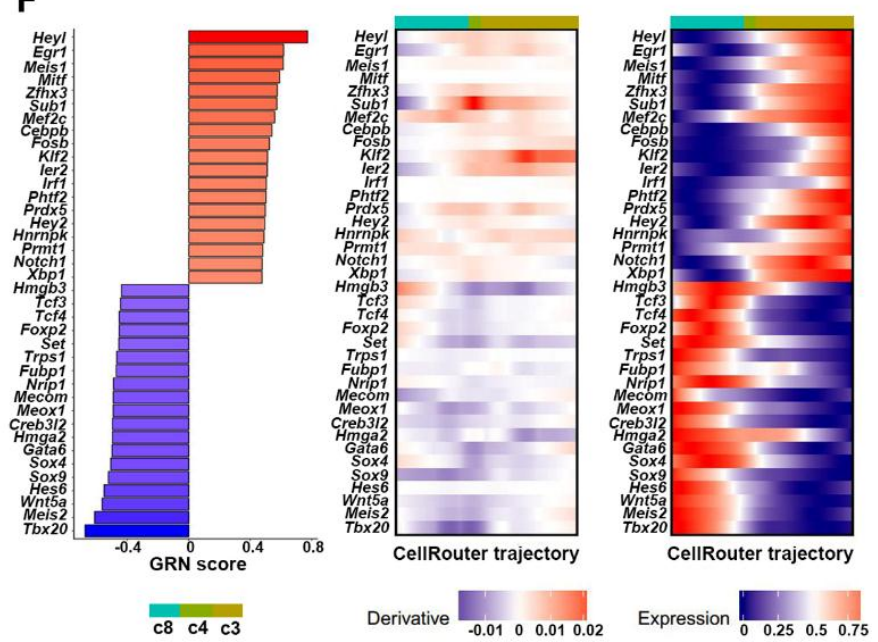

Figure 5 
bioRxiv preprint doi: https://doi.org/10.1101/469346; this version posted November 13, 2018. The copyright holder for this preprint (which was not certified by peer review) is the author/funder, who has granted bioRxiv a license to display the preprint in perpetuity. It is made available under aCC-BY-NC-ND 4.0 International license.

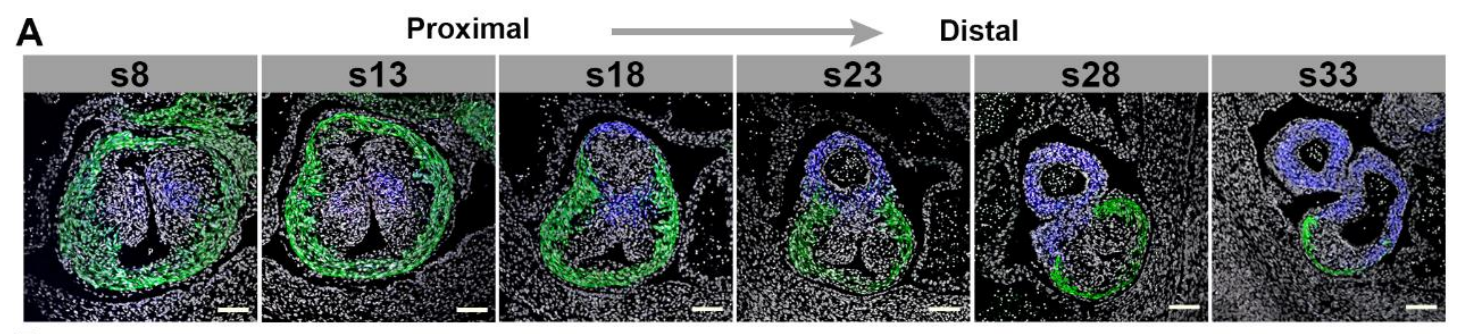

B
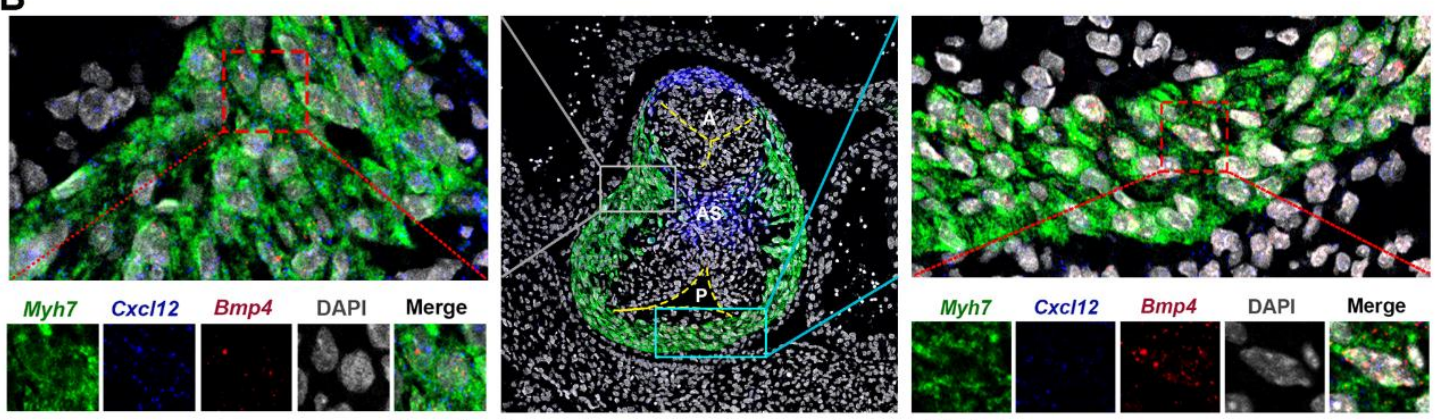

c

Proximal

Distal
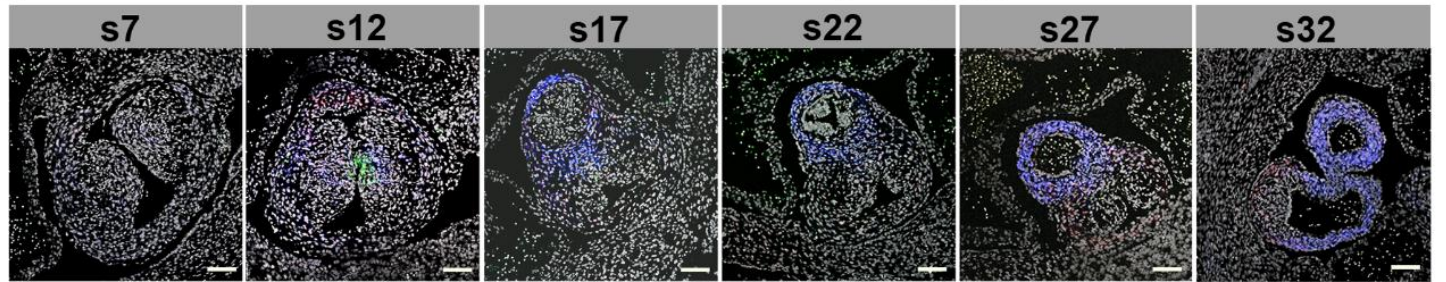

D
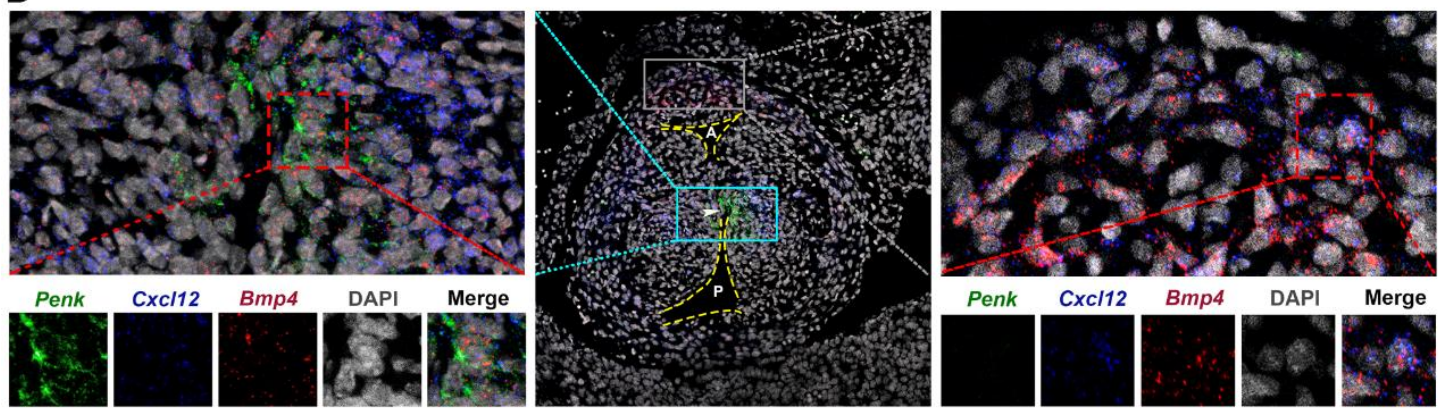

Figure 6 